\title{
Unusual Acute Onset of Symptomatic Langerhans Cell Histiocytosis of the Skull: Case Report and Literature Review
}

\author{
Paul E. Kaloostian ${ }^{1}$, Han Chen $^{2 *}$, Franklin Westhout ${ }^{2}$, Erich Marchand ${ }^{2}$ \\ ${ }^{1}$ Johns Hopkins University Medical Center, Baltimore, USA \\ ${ }^{2}$ Department of Neurosurgery, University of New Mexico, Albuquerque, USA \\ Email: "pkaloos1@jhmi.edu
}

Received March 19, 2013; revised April 18, 2013; accepted May 2, 2013

Copyright (C) 2013 Paul E. Kaloostian et al. This is an open access article distributed under the Creative Commons Attribution License, which permits unrestricted use, distribution, and reproduction in any medium, provided the original work is properly cited.

\begin{abstract}
Introduction: Langerhans cell histiocytosis (LCH) is a group of idiopathic disorders characterized by the proliferation of specialized bone marrow-derived Langerhans cells (LCs) and mature eosinophils, resulting in solitary or few, indolent and chronic, lesions of bone or other organs called eosinophilic granulomas. Calvarial LCH is quite rare and an underappreciated differential etiology of skull lesions. We present a most unusual case of a young child with hyperacutely symptomatic langerhans histiocytosis of the skull. Method: A 7-year-old male presented with a history of increasing (progressive) frontal headaches of 8 days duration, unaccompanied by associated nausea, vomiting, or diplopia. His only additional complaint was a hard bump on his forehead. MRI and CT done in the ER identified a right fronto-parietal lesion with associated skull erosion. Nuclear medicine and SPECT studies confirmed an erosive skull lesion without significant metabolic activity. A right frontal craniectomy and excision was performed. Results: A soft, rubbery well-circumcised mass coming from the diploic layer of the skull with involvement of bone was identified. The mass had eroded both the outer and inner table of the skull, and the involved area of the right frontal bone was resected. Intra-operative histo-pathologic analysis of the lesion revealed Langerhans cell histiocytosis without involvement of the dura. The patient experienced no neurological worsening as a result of the resection. He was discharged home in stable condition. Conclusion: LCH lesions of the skull are common findings, however, this focal hyperacute symptomatic presentation is most rare and should not deter us from anticipating an erosive bony tumor and planning timely surgical management.
\end{abstract}

Keywords: Langerhans Cell Histiocytosis; Histiocytosis X; Letterer Siwe; Eosinophilic Granuloma; Skull Neoplasms

\section{Introduction}

Skull inner mass lesions include metastatic cancer [1] (leiomyosarcoma, leiomyoma) [2], sarcomas (rhabdomyosarcoma, etc.), meningeal lesions (meningioma) and Langerhans cell histiocytosis. Langerhans cell histiocytosis is a type of neoplasm characterized by idiopathic histiocytic and eosinophilic proliferation [3]. The name reflects the primary cell involved as well as the pathophysiology of the disease. The three subtypes are Letterer-Siwe disease, Hand-Schüller-Christian disease and eosinophilic granuloma [3,4]. Letterer-Siwe disease is a diffuse, systemic form of the disease that is uniformly fatal within 1 - 2 years. Eosinophilic granuloma is

${ }^{*}$ Corresponding author. a localized form of the disease and usually presents as a unifocal bony lesion, typically involving the temporal or frontal bone. The tumor is further characterized by a local collection of histiocytes and eosinophils that stimulate bone resorption, producing a radiolucent lesion. Diagnosis is confirmed by open biopsy. The ultra-structural hallmark on histopathological analysis is Birbeck granules [5]. High resolution computed tomography (HRCT) reveals "punched-out" defects or "moth-eaten" lesions. [(Briggs RD, Gadre A.Differential Diagnosis of Temporal Bone and Skull Base Lesions. Grand Rounds Presentation, UTMB, Dept. of Otolaryngology, December 19, 2001)] Hand-Schuller-Christian disease is a systemic variant of eosinophilic granuloma [5]. There are typically multiple punched-out defects in the temporal bone, 
often bilateral, clinically accompanied by chronic otorrhea [6]. The disease may affect other organs including the abdominal viscera and skin. The onset of symptoms is typically several weeks to months. We present a rare case of Langerhans cell histiocytosis with a relatively short time of onset.

\section{Case Report}

History and examination: A 7 years old male presented with an 8-day history of intermittent, progressively worsening occipital headaches. This was also accompanied by right jaw pain, intermittent nausea without emesis, and abdominal pain. He had no significant past medical history and had never complained of headaches previously. In the three days prior to presentation his right parietal scalp had been noted to be tender. A CT scan obtained by the referring physician revealed a right temporal skull defect, likely a soft tissue mass with skull invasion. On physical exam there were no focal neurological deficits. A skeletal survey demonstrated no other osseous lesions (Figures 1(a) and (b)).

Imaging: MRI demonstrated a (Figures 1(c) and (d)) right lateral frontotemporal extracranial soft tissue swelling ovoid in appearance, with an approximately 1.6 cm cystic region with an approximately $1.5 \mathrm{~cm}$ adjacent skull defect involving the inner and outer tables. Also noted were edema within the regional diploe and reactive dural contrast enhancement regionally. At this point an infectious or neoplastic etiology could yet not be ruled out. A nuclear medicine study was performed in which MDP distribution showed vague, increased uptake corresponding to the right temporal bone lesion as seen on the CT. This was confirmed with SPECT imaging of the head (6.6 mCi of Technetium-99 m MDP, IV) (Figure 2).

We performed a right-sided craniectomy, greater than 5 centimeters, for removal of this skull tumor. A soft, rubbery well-circumcised mass coming from the diploic layer of the skull was identified with involvement of bone approximately 5 centimeters in width. The texture was very soft and rubbery with no purulent material. We resected this mass and noticed that the diploic layer of the skull had been very soft and invaded multiple levels, and so we used a drill and performed our craniectomy until we identified normal bone throughout. The dura was not invaded. We performed a cranioplasty with OsteoMed $^{\circledR}$ (OsteoMed, 3885 Arapaho Road, Addison, TX 75001) mesh and screws, approximately 5 centimeters in size.

Pathological findings: Histopathological confirmation analysis revealed Langerhans Cell Histiocytosis. Immunohistochemistry revealed cells positive for S100, CD1a, Langerin and CD 68, consistent with a diagnosis of LCH.

Postoperative course: The patient tolerated the procedure and was discharged home in good condition.

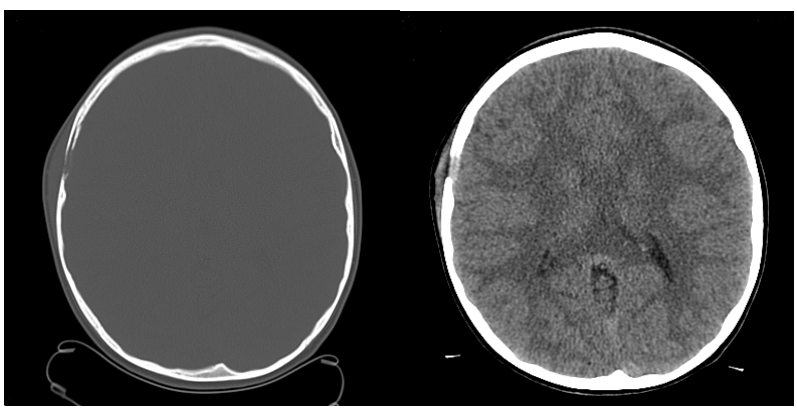

(a)

(b)

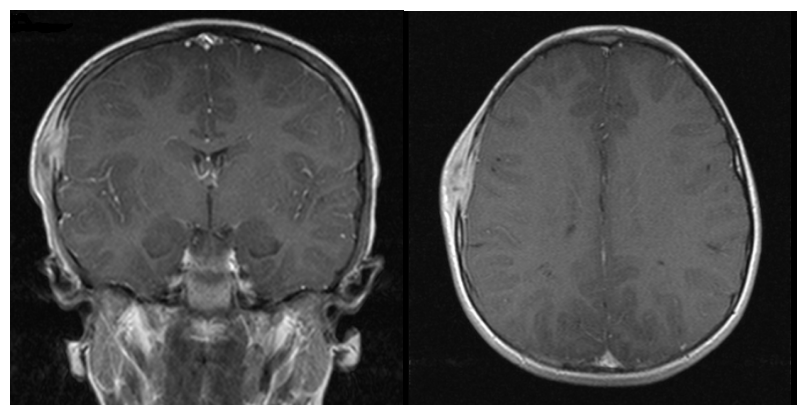

(c)

(d)

Figure 1. (a), (b): CT of the head demonstrating a soft tissue with lysis of the underlying skull. The bone windows (a) clearly demonstrate the involvement of the inner and outer tables. (c), (d): Coronal and axial MRI demonstrating a lateral right frontotemporal extracranial soft tissue swelling (with an approximately $1.6 \mathrm{~cm}$ ovoid cystic region) associated with an approximately $1.5 \mathrm{~cm}$ adjacent skull defect involving the inner and outer tables, edema within the regional diploe, and reactive dural contrast enhancement regionally. No other areas of abnormal contrast enhancement were identified intracranially. No other calvarial abnormalities are noted. The mastoid air cells, paranasal sinuses and orbits were unremarkable, as were the remaining extracranial soft tissues.

\section{Discussion}

The precise causes of most histiocytoses are not known, but infections, particularly viral [7], cellular and immune dysfunctions [8] have been implicated in the etiology and pathophysiology of this disorder. Källén et al., reported an increased rate of diagnosed histiocytosis in children conceived by means of in vitro fertilization, 6 cases of Langerhans histiocytosis against 1.0 expected [9].

Eosinophilic granuloma is the most common form of $\mathrm{LCH}$, and usually presents as a solitary osteolytic lesion of the skull or spine. Hand-Schuller-Christian disease presents with multi-focal LCH lesions of the bone with hypothalamic involvement [5], and this multi focal or disseminated $\mathrm{LCH}$ is responsible for most cases with CNS involvement. Abt-Letterer-Siwe disease is characterized by skin, lymph nodes and visceral involvement, rarely presenting with CNS involvement [5].

Based on this classification, the patient in our case likely presented with Hand-Schüller-Christian (HSC) 


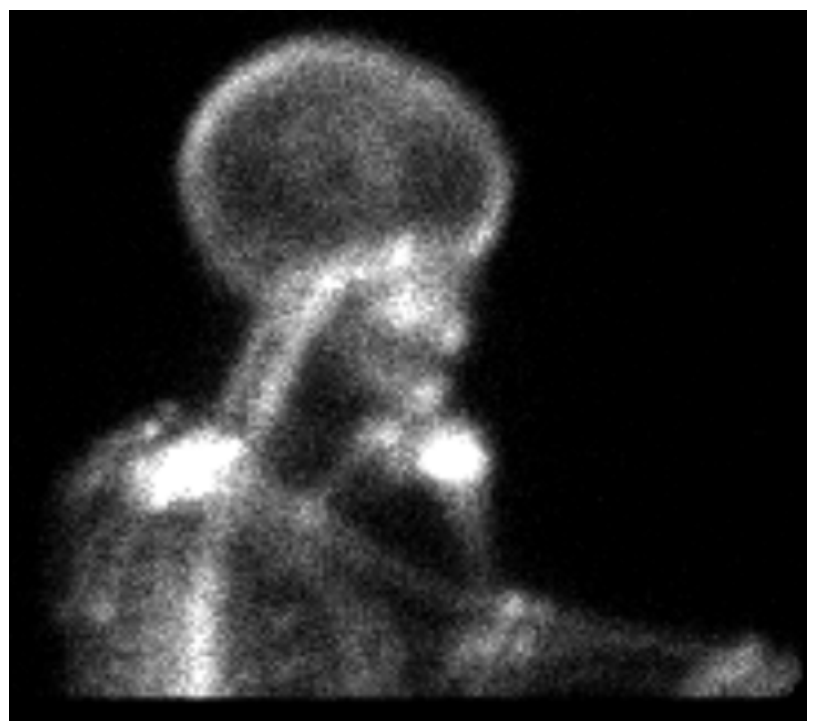

Figure 2. NM: The $(6.6 \mathrm{mCi}$ of technetium $99 \mathrm{~m}$ (99 mTc) methylene diphosphonate (MDP) IV) distribution shows vague, increased uptake corresponding to the right temporal bone lesion seen on CT. This was confirmed with SPECT imaging of the head. There were no other concerning abnormalities.

sub-classification of LCH as opposed to eosinophilic granuloma. That is an important observation as it relates to the morbidity of his LCH. Infiltration of the CNS can cause location dependent signs and symptoms usually due to compression of dural sinuses and cranial nerves [1]. Disruptions of hypothalamic and pituitary function are most commonly observed. This would include diabetes insipidus related symptoms and, to a lesser extent, growth-hormone deficiency and hypopituitarism, and other hormonal imbalances [11]. Other symptoms, such as seizures and those related to mass effect and increased intracranial pressure depend on the site and volume of the space-occupying lesion.

Initial growth of calvarial LCH has been reported as fast, plateaus in adults, and may even spontaneously resolve [12]. Patients with skull metastases tend to present with a shorter history of symptoms. Stark et al. found an average duration of symptoms of 2 months for skull metastases vs. 18 months for primary skull lesions [1]. Calvarial eosinophilic granuloma has been reported to spontaneously resolve in 4 out of 17 children with a tender calvarial mass [12]. These masses had enlarged rapidly over days and resolved within weeks, with no recurrence at follow-up from 2 months up to 7 years. These authors suggest that observation can be considered in children with solitary calvarial masses with radiographic features of EG, with sufficient delay in surgical intervention to allow time for spontaneous resolution [12]. However, surgery remains the best treatment [12-14] allowing for accurate diagnosis with tissue biopsy. Considering these observations, it is clear that the presentation of primary LCH over an 8 days period is unusual.

Fung et al. described a LCH developing in a 29 years old woman over a relatively short duration of two months [15]. She presented with an enlarging lesion over her left frontal bone following minor trauma. CT scan showed an osteolytic lesion with an overlying soft tissue mass, thought to be an unhealed skull fracture with pseudomeningocele [15]. Even though most LCH lesions are of soft-solid consistency, fluid filled LCH lesion of the calvarium has been described [16].

The relative frequency of occurrence on the cranial vault is (65\%), in the suprasellar region (21\%) and in the spinal column $(8 \%)[14,17]$. Unifocal involvement of the frontal bone is rare [18]. Involvement of the Clivus is rare, Brisman et al. reported 3 out of 87 cases with clival involvement [21], who underwent treatment with Stereotactic Radiosurgery with resolution. Less frequently isolated CNS lesions tend to occur in the temporal lobe [13], and pituitary-hypothalamic axis $[14,19]$.

Cranioplasty of the skull after excision is usually accomplished using titanium mesh plates and cranioplasty with hydroxyapatite [20]. Optional treatments have included stereotactic radiosurgery (SRS) [4] and intralesional steroid therapy [10]. Etoposide (VP16), is a semisynthetic epipodophyllotoxin derivative effective in the treatment of malignancies of the monocyte-macrophage lineage and used in resistant or relapsed childhood LCH [22].

The duration of disease can vary widely, however, it can be relatively short, as measured in days, as illustrated in the case presented. A thorough history and physical and total CNS imaging, including [high resolution] CT scans of the head and complete spine as well as MR imaging with a pituitary protocol would be recommended to rule out CNS and systemic disease. Hormonal aberrations can be present with systemic involvement and most commonly presents as diabetes insipidus. In the absence of systemic disease, there are usually no neurological deficits or upper motor neuron findings in skull-based Langerhans Cell Histiocytosis, or Eosinophilic Granuloma, but CNS involvement is common in HSC. Certainly, LCH should not be overlooked but included in the working differential for lesions of the skull that are enlarging and tender.

\section{REFERENCES}

[1] A. M. Stark, T. Eichman and H. M. Mehdorn, "Skull Metastases: Clinical Features, Differential Diagnosis, and Review of the Literature," Surgical Neurology, Vol. 60, No. 3, 2003, pp. 219-225. doi:10.1016/S0090-3019(03)00269-6

[2] G. Alessi, M. Lemmerling, L. Vereecken and L. De Waele, "Benign Metastasizing Leiomyoma to Skull Base and Spine: A Report of Two Cases," Clinical Neurology and Neurosurgery, Vol. 105, No. 3, 2003, pp. 170-174. 


\section{doi:10.1016/S0303-8467(03)00002-7}

[3] J. McLelland, J. Pritchard and A. C. Chu, "Current Controversies: Histiocytosis-X,” Hematology/Oncology Clinics of North America, Vol. 1, No. 1, 1987, pp. 147-162.

[4] A. Shahla, V. Parvaneh and H. D. Hossein, "Langerhans Cells Histiocytosis in One Family," Pediatric Hematology-Oncology, Vol. 21, No. 4, 2004, pp. 313-320. doi:10.1080/08880010490440446

[5] P. Kleihaus and W. K. Cavenee, "Pathology and Genetics: Tumours of the Nervous System-WHO Classification of Tumours, International Agency for Research on Cancer,” IARC Press, Lyon, 2000, p. 204.

[6] C. Hadjigeorgi, C. Parpounas, P. Zarmakoupis and S. Lafoyianni, "Eosinophilic Granuloma of the Temporal Bone: Radiological Approach in the Pediatric Patient," Pediatric Radiology, Vol. 20, No. 7, 1990, pp. 546-549. doi:10.1007/BF02011387

[7] M. A. Leahy, S. M. Krejci, M. Friednash, S. S. Stockert, H. Wilson, J. C. Huff, et al., "Human Herpesvirus 6 Is Present in Lesions of Langerhans Cell Histiocytosis," Journal of Investigative Dermatology, Vol. 101, 1993, pp. 642-645. doi:10.1111/1523-1747.ep12371669

[8] C. L. Willman, L. Busque, B. B. Griffith, B. E. Favara, K. L. McClain, M. H. Duncan, et al., 'Langerhans' Cell Histiocytosis (Histiocytosis-X)-A Clonal Proliferative Disease," The New England Journal of Medicine, Vol. 331, 1994, pp. 154-160. doi:10.1056/NEJM199407213310303

[9] B. Källén, O. Finnström, A. Lindam, E. Nilsson, K. G. Nygren and P. O. Olausson, "Cancer Risk in Children and Young Adults Conceived by in Vitro Fertilization,” Pediatrics, Vol. 126, 2010, pp. 270-276. doi:10.1542/peds.2009-3225

[10] J. D. Wirtschafter, M. Nesbit, P. Anderson and K. McClain, "Intralesional Methylprednisolone for Langerhans' Cell Histiocytosis of the Orbit and Cranium," Journal of Pediatric Ophthalmology and Strabismus, Vol. 24, No. 4, 1987, pp. 194-197.

[11] G. A. Kaltsas, T. B. Powles, J. Evanson, P. N. Plowman, J. E. Drinkwater, P. J. Jenkins, et al., "Hypothalamo-Pituitary Abnormalities in Adult Patients with Langerhans Cell Histiocytosis: Clinical, Endocrinological, and Radiological Features and Response to Treatment," The Journal of Clinical Endocrinology \& Metabolism, Vol. 85, No. 4, 2000, pp. 1370-1376. doi:10.1210/jc.85.4.1370

[12] M. Oliveira, P. Steinbok, J. Wu, N. Heran and D. Cochrane, "Spontaneous Resolution of Calvarial Eosinophilic
Granuloma in Children,” Pediatric Neurosurgery, Vol. 38, 2003, pp. 247-252. doi:10.1159/000069828

[13] M. J. Katati, J. M. Martin, J. Pastor and V. Arjona, "Isolated Primary Langerhans' Cell Histiocytosis of Central Nervous System,” Neurocirugía, Vol. 13, No. 6, 2002, pp. 477-478.

[14] D. Belen, A. Colak and O. E. Ozcan, "CNS Involvement of Langerhans Cell Histiocytosis: Report of 23 Surgically Treated Cases,” Neurosurgical Review, Vol. 19, No. 4, 1996, pp. 247-252. doi:10.1007/BF00314840

[15] K. M. Fung, J. M. Schwalb, H. A. Riina, J. S. Kurana, J. M. Mindaxy, M. S. Grady, et al., "February 2002: 29-Year-Old Woman with a Skull Mass for 2 Months," Brain Pathology, Vol. 12, No. 3, 2002, pp. 393-394.

[16] A. Taranath and E. Onikul, "Fluid-Fluid Levels in Transcalvarial Langerhans' Cell Histiocytosis,” Pediatric Radiology, Vol. 34, No. 4, 2004, p. 363. doi:10.1007/s00247-003-1134-z

[17] V. Debois and R. van den Bergh, "Benign Tumors of the Cranial Vault, a Report of 12 Cases,” Clinical Neurology and Neurosurgery, Vol. 81, No. 1, 1979, pp. 1-12. doi:10.1016/S0303-8467(79)80002-5

[18] S. I. Basha, J. B. Kinsella and S. S. Napier, "Unifocal Langerhans' Cell Histiocytosis and Frontal Sinus Agenesis: Report of a Rare Case,” Ear, Nose \& Throat Journal, Vol. 81, No. 11, 2002, pp. 795-799.

[19] A. Isoo, K. Ueki, T. Ishida, T. Yoshikawa, T. Fujimaki, I. Suzuki, et al., "Langerhans Cell Histiocytosis Limited to the Pituitary-Hypothalamic Axis-Two Case Reports,” Neurologia Medico-Chirurgica, Vol. 40, No. 10, 2000, pp. 532-535. doi:10.2176/nmc.40.532

[20] L. I. Malis, "Titanium Mesh and Acrylic Cranioplasty," Neurosurgery, Vol. 25, No. 3, 1989, pp. 351-355. doi:10.1227/00006123-198909000-00005

[21] J. L. Brisman, N. A. Feldstein, N. J. Tarbell, D. Cohen, A. L. Cargan, J. Haddad Jr. and J. N. Bruce, "Eosinophilic Granuloma of the Clivus: Case Report, Follow-Up of Two Previously Reported Cases, and Review of the Literature on Cranial Base Eosinophilic Granuloma,” Neurosurgery, Vol. 41, No. 1, 1997, pp. 273-278. doi:10.1097/00006123-199707000-00048

[22] S. C. Mayou, A. C. Chu, D. D. Munro and N. Plowman, "Langerhans-Cell Histiocytosis-Excellent Response to Etoposide,” Clinical and Experimental Dermatology, Vol. 16, No. 4, 1991, pp. 292-294. doi:10.1111/j.1365-2230.1991.tb00379.x

\section{Abbreviations}

$$
\begin{aligned}
& \text { CT = Computed Tomography } \\
& \text { CNS = Central nervous system } \\
& \text { LCH = Langerhans Cell Histiocytosis } \\
& \text { LC = Langerhans Cell }
\end{aligned}
$$

\author{
MRI/MR = Magnetic Resonance Imaging \\ MDP = methylene diphosphonate \\ $\mathrm{NM}=$ Nuclear Medicine \\ SPECT = Single-photon emission computed tomo-
} graphy 